\title{
Product of operators and numerical range preserving maps
}

\author{
by \\ ChI-Kwong Li (Williamsburg, VA) and Nung-Sing Sze (Hong Kong)
}

To Professor Miroslav Fiedler on the occasion of his 80th birthday

\begin{abstract}
Let $\mathbf{V}$ be the $C^{*}$-algebra $B(H)$ of bounded linear operators acting on the Hilbert space $H$, or the Jordan algebra $S(H)$ of self-adjoint operators in $B(H)$. For a fixed sequence $\left(i_{1}, \ldots, i_{m}\right)$ with $i_{1}, \ldots, i_{m} \in\{1, \ldots, k\}$, define a product of $A_{1}, \ldots, A_{k} \in \mathbf{V}$ by $A_{1} * \cdots * A_{k}=A_{i_{1}} \cdots A_{i_{m}}$. This includes the usual product $A_{1} * \cdots * A_{k}=A_{1} \cdots A_{k}$ and the Jordan triple product $A * B=A B A$ as special cases. Denote the numerical range of $A \in \mathbf{V}$ by $W(A)=\{(A x, x): x \in H,(x, x)=1\}$. If there is a unitary operator $U$ and a scalar $\mu$ satisfying $\mu^{m}=1$ such that $\phi: \mathbf{V} \rightarrow \mathbf{V}$ has the form
\end{abstract}

$$
A \mapsto \mu U^{*} A U \quad \text { or } \quad A \mapsto \mu U^{*} A^{t} U,
$$

then $\phi$ is surjective and satisfies

$$
W\left(A_{1} * \cdots * A_{k}\right)=W\left(\phi\left(A_{1}\right) * \cdots * \phi\left(A_{k}\right)\right) \quad \text { for all } A_{1}, \ldots, A_{k} \in \mathbf{V} .
$$

It is shown that the converse is true under the assumption that one of the terms in $\left(i_{1}, \ldots, i_{m}\right)$ is different from all other terms. In the finite-dimensional case, the converse can be proved without the surjectivity assumption on $\phi$. An example is given to show that the assumption on $\left(i_{1}, \ldots, i_{m}\right)$ is necessary.

1. Introduction. Let $H$ be a Hilbert space having dimension at least 2 . Denote by $B(H)$ the $C^{*}$-algebra of bounded linear operators acting on $H$, and $S(H)$ the Jordan algebra of self-adjoint operators in $B(H)$. If $H$ has dimension $n<\infty$, then $B(H)$ is identified with the algebra $M_{n}$ of $n \times n$ complex matrices and $S(H)$ is identified with the set $S_{n}$ of $n \times n$ complex Hermitian matrices. Define the numerical range of $A \in B(H)$ by

$$
W(A)=\{(A x, x): x \in H,(x, x)=1\} .
$$

2000 Mathematics Subject Classification: 47A12, 47B15, 47B49, 15A60, 15A04, 15A18.

Key words and phrases: numerical range, Jordan triple product.

$\mathrm{Li}$ is an honorary professor of the Heilongjiang University and an honorary professor of the University of Hong Kong. His research was partially supported by a USA NSF grant and a HK RCG grant.

The research of Sze was partially supported by a HKU studentship and a HK RGC grant. He would like to thank Dr. Jor-Ting Chan for his guidance and encouragement. 
Let $U \in B(H)$ be a unitary operator, and define a mapping $\phi$ on $B(H)$ or $S(H)$ by

$$
A \mapsto U^{*} A U \quad \text { or } \quad A \mapsto U^{*} A^{t} U,
$$

where $A^{t}$ is the transpose of $A$ with respect to a fixed orthonormal basis. (We will always use this interpretation of $A^{t}$ in our discussion.) Then $\phi$ is a bijective linear map preserving the numerical range, i.e., $W(\phi(A))=W(A)$ for all $A$.

There has been considerable interest in studying the converse of the above statement. Pellegrini [8] obtained an interesting result on numerical range preserving maps on a general $C^{*}$-algebra, which implies that a surjective linear map $\phi: B(H) \rightarrow B(H)$ preserving the numerical range must be of the above form. Furthermore, by the result in [7], the same conclusion also holds for linear maps $\phi$ acting on $S(H)$. In [6], the author showed that additive preservers of the numerical range of matrices must be linear and have the standard form $A \mapsto U^{*} A U$ or $A \mapsto U^{*} A^{t} U$. In [2], it was shown that a multiplicative map $\phi: M_{n} \rightarrow M_{n}$ satisfies $W(\phi(A))=W(A)$ for all $A \in M_{n}$ if and only if $\phi$ has the form $A \mapsto U^{*} A U$ for some unitary matrix $U \in M_{n}$. In [5], the authors replaced the condition that " $\phi$ is multiplicative and preserves the numerical range" on the surjective map $\phi: B(H) \rightarrow B(H)$

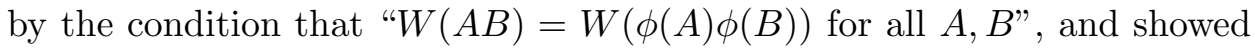
that such a map has the form $A \mapsto \pm U^{*} A U$ for some unitary operator $U \in B(H)$. They also showed that a surjective map $\phi: B(H) \rightarrow B(H)$ satisfies $W(A B A)=W(\phi(A) \phi(B) \phi(A))$ for all $A, B \in B(H)$ if and only if $\phi$ has the form $A \mapsto \mu U^{*} A U$ or $A \mapsto \mu U^{*} A^{t} U$ for some unitary operator $U \in B(H)$ and $\mu \in \mathbb{C}$ with $\mu^{3}=1$. Similar results for mappings on $S(H)$ were also obtained. Recently, Gau and $\mathrm{Li}[3]$ obtained a similar result for surjective maps $\phi: \mathbf{V} \rightarrow \mathbf{V}$, where $\mathbf{V}=B(H)$ or $S(H)$, preserving the numerical range of the Jordan product, i.e., $W(A B+B A)=W(\phi(A) \phi(B)+\phi(B) \phi(A))$ for all $A, B \in \mathbf{V}$. Specifically, they showed that such a map must be of the form $A \mapsto \pm U^{*} A U$ or $A \mapsto \pm U^{*} A^{t} U$ for some unitary operator $U \in B(H)$. Moreover, the surjectivity assumption can be removed in the finite-dimensional case.

It is interesting that all the results mentioned in the preceding paragraph illustrate that under some mild assumptions, a numerical range preserving map $\phi$ is a $C^{*}$-isomorphism on $B(H)$ or a Jordan isomorphism on $S(H)$ up to a scalar multiple. Following this line of study, we consider a product of matrices involving $k$ matrices with $k \geq 2$ which includes the usual product $A_{1} * \cdots * A_{k}=A_{1} \cdots A_{k}$, and the Jordan triple product $A * B=A B A$. We prove the following result.

Theorem 1.1. Let $(\mathbb{F}, \mathbf{V})=(\mathbb{C}, B(H))$ or $(\mathbb{R}, S(H))$. Fix a positive integer $k$ and a finite sequence $\left(i_{1}, \ldots, i_{m}\right)$ such that $\left\{i_{1}, \ldots, i_{m}\right\}=\{1, \ldots, k\}$ 
and there is an $i_{r}$ not equal to $i_{s}$ for all other $s$. For $A_{1}, \ldots, A_{k} \in \mathbf{V}$, let

$$
A_{1} * \cdots * A_{k}=A_{i_{1}} \cdots A_{i_{m}} .
$$

$A$ surjective map $\phi: \mathbf{V} \rightarrow \mathbf{V}$ satisfies

$$
W\left(\phi\left(A_{1}\right) * \cdots * \phi\left(A_{k}\right)\right)=W\left(A_{1} * \cdots * A_{k}\right) \quad \text { for all } A_{1}, \ldots, A_{k} \in \mathbf{V}
$$

if and only if there exist a unitary operator $U \in B(H)$ and a scalar $\mu \in \mathbb{F}$ with $\mu^{m}=1$ such that one of the following conditions holds:

(a) $\phi$ has the form $A \mapsto \mu U^{*} A U$.

(b) $r=(m+1) / 2,\left(i_{1}, \ldots, i_{m}\right)=\left(i_{m}, \ldots, i_{1}\right)$, and $\phi$ has the form $A \mapsto$ $\mu U^{*} A^{t} U$.

(c) $\mathbf{V}=S_{2},\left(i_{r+1}, \ldots, i_{m}, i_{1}, \ldots, i_{r-1}\right)=\left(i_{r-1}, \ldots, i_{1}, i_{m}, \ldots, i_{r+1}\right)$ and $\phi$ has the form $A \mapsto \mu U^{*} A^{t} U$.

Here $A^{t}$ denotes the transpose of $A$ with respect to a certain orthonormal basis of $H$. Furthermore, if the dimension of $H$ is finite, then the surjectivity assumption on $\phi$ can be removed.

Note that the assumption that there is $i_{r} \notin\left\{i_{1}, \ldots, i_{r-1}, i_{r+1}, \ldots, i_{m}\right\}$ is necessary. For example, if $A * B=A B B A$, then mappings $\phi$ satisfying $W(\phi(A) * \phi(B))=W(A * B)$ may not have nice structure. For instance, $\phi$ can send all involutions, i.e., those operators $X \in B(H)$ such that $X^{2}=I_{H}$, to a fixed involution, and $\phi(X)=X$ for other $X$.

For the usual product $A_{1} * \cdots * A_{k}=A_{1} \cdots A_{k}$ and the Jordan triple product $A * B=A B A$, Hou and Di [5] have also obtained the result for $B(H)$ in Theorem 1.1 with the surjectivity assumption. Our result is stronger when $H$ is finite-dimensional.

It turns out that Theorem 1.1 can be deduced from the following special case.

TheOREM 1.2. Let $(\mathbb{F}, \mathbf{V})=(\mathbb{C}, B(H))$ or $(\mathbb{R}, S(H))$. Suppose $r, s$ and $m$ are nonnegative integers such that $m-1=r+s>0$. A surjective map $\phi: \mathbf{V} \rightarrow \mathbf{V}$ satisfies

$$
W\left(\phi(A)^{r} \phi(B) \phi(A)^{s}\right)=W\left(A^{r} B A^{s}\right) \quad \text { for all } A, B \in \mathbf{V}
$$

if and only if there exist a unitary operator $U \in B(H)$ and a scalar $\mu \in \mathbb{F}$ with $\mu^{m}=1$ such that one of the following conditions holds:

(a) $\phi$ has the form $A \mapsto \mu U^{*} A U$.

(b) $r=s$ and $\phi$ has the form $A \mapsto \mu U^{*} A^{t} U$.

(c) $\mathbf{V}=S_{2}$ and $\phi$ has the form $A \mapsto \mu U^{*} A^{t} U$.

Here $A^{t}$ denotes the transpose of $A$ with respect to a certain orthonormal basis of $H$. Furthermore, if the dimension of $H$ is finite, then the surjectivity assumption on $\phi$ can be removed. 
We present some auxiliary results in Section 2, and the proofs of the theorems in Section 3.

2. Auxiliary results. For any $x, y \in H$, denote by $x y^{*}$ the rank one operator $\left(x y^{*}\right) z=(z, y) x$ for all $z \in H$. Then for any operator $A \in B(H)$ with finite rank, $A$ can be written as $x_{1} y_{1}^{*}+\cdots+x_{k} y_{k}^{*}$ for some $x_{i}, y_{i} \in H$. Define the trace of $A$ by

$$
\operatorname{tr}(A)=\left(x_{1}, y_{1}\right)+\cdots+\left(x_{k}, y_{k}\right) .
$$

If $H$ is finite-dimensional, $\operatorname{tr}(A)$ is equivalent to the usual matrix trace, i.e., the sum of all diagonal entries of the matrix $A$. For each positive integer $m$, let

$$
\mathcal{R}^{m}=\left\{\mu x x^{*}: \mu \in \mathbb{F} \text { and } x \in H \text { with }(x, x)=1=\mu^{m}\right\} .
$$

Note that $\mathcal{R}^{1}$ is the set of Hermitian rank one idempotents, and for all $m>1, \mathcal{R}^{1} \subseteq \mathcal{R}^{m}$.

Proposition 2.1. Let $\mathbf{V}=B(H)$ or $S(H)$ and $\mathbb{F}=\mathbb{C}$ or $\mathbb{R}$ accordingly. Suppose $m$ is a positive integer with $m>1$, and $\phi: \mathbf{V} \rightarrow \mathbf{V}$ is a map satisfying

$$
\operatorname{tr}\left(\phi(A)^{m-1} \phi(B)\right)=\operatorname{tr}\left(A^{m-1} B\right) \quad \text { for all } A \in \mathcal{R}^{m} \text { and } B \in \mathbf{V} .
$$

If $H$ is finite-dimensional, then $\phi$ is an invertible $\mathbb{F}$-linear map. If $H$ is infinite-dimensional and $\phi\left(\mathcal{R}^{m}\right)=\mathcal{R}^{m}$, then $\phi$ is $\mathbb{F}$-linear.

Proof. Suppose $H$ is finite-dimensional. We use an argument similar to that in the proof of Proposition 1.1 in [1]. Let $\mathbf{V}=M_{n}$ or $S_{n}$. For every $X=\left(x_{i j}\right) \in \mathbf{V}$, let $R_{X}$ be the $n^{2}$ row vector

$$
R_{X}=\left(x_{11}, \ldots, x_{1 n}, x_{21}, \ldots, x_{2 n}, \ldots, x_{n 1}, \ldots, x_{n n}\right),
$$

and $C_{X}$ the $n^{2}$ column vector

$$
C_{X}=\left(x_{11}, x_{21} \ldots, x_{n 1}, x_{12}, \ldots, x_{n 2}, \ldots, x_{1 n}, \ldots, x_{n n}\right)^{t} .
$$

Then we deduce from (2.1) that for all $A \in \mathcal{R}^{m}$ and $B \in \mathbf{V}$,

$$
R_{\phi(A)^{m-1}} C_{\phi(B)}=\operatorname{tr}\left(\phi(A)^{m-1} \phi(B)\right)=\operatorname{tr}\left(A^{m-1} B\right)=R_{A^{m-1}} C_{B} .
$$

Note that we can choose $A_{1}, \ldots, A_{n^{2}}$ in $\mathcal{R}^{m}$ such that $\left\{A_{1}^{m-1}, \ldots, A_{n^{2}}^{m-1}\right\}$ forms a basis for $\mathbf{V}$. Let $\Delta$ and $\Delta_{\phi}$ be $n^{2} \times n^{2}$ matrices having rows $R_{A_{1}^{m-1}}, \ldots, R_{A_{n^{2}}^{m-1}}$ and $R_{\phi\left(A_{1}\right)^{m-1}}, \ldots, R_{\phi\left(A_{n^{2}}\right)^{m-1}}$, respectively. By $(2.2)$,

$$
\Delta_{\phi} C_{\phi(B)}=\Delta C_{B} \text { for all } B \in \mathbf{V} .
$$

Now take a basis $\left\{B_{1}, \ldots, B_{n^{2}}\right\}$ in $\mathbf{V}$ and let $\Omega$ and $\Omega_{\phi}$ be the $n^{2} \times n^{2}$ matrices having columns $C_{B_{1}}, \ldots, C_{B_{n^{2}}}$ and $C_{\phi\left(B_{1}\right)}, \ldots, C_{\phi\left(B_{n^{2}}\right)}$, respectively. Then $\Delta_{\phi} \Omega_{\phi}=\Delta \Omega$. Note that both $\Delta$ and $\Omega$ are invertible, hence so is $\Delta_{\phi}$. 
Therefore, for any $B \in \mathbf{V}$,

$$
C_{\phi(B)}=\Delta_{\phi}^{-1} \Delta C_{B}
$$

Hence, $\phi$ is invertible and $\mathbb{F}$-linear.

Next, suppose $H$ is infinite-dimensional and $\phi\left(\mathcal{R}^{m}\right)=\mathcal{R}^{m}$. Take any $X, Y \in \mathbf{V}$. For any $x \in H$ with $(x, x)=1$, since $\mathcal{R}^{1} \subseteq \mathcal{R}^{m}=\phi\left(\mathcal{R}^{m}\right)$, there is $A \in \mathcal{R}^{m}$ such that $\phi(A)=x x^{*}$. Then $\phi(A)^{m-1}=x x^{*}$ and

$$
\begin{aligned}
(\phi(X+Y) x, x) & =\operatorname{tr}\left(x x^{*} \phi(X+Y)\right)=\operatorname{tr}\left(\phi(A)^{m-1} \phi(X+Y)\right) \\
& =\operatorname{tr}\left(A^{m-1}(X+Y)\right)=\operatorname{tr}\left(A^{m-1} X\right)+\operatorname{tr}\left(A^{m-1} Y\right) \\
& =\operatorname{tr}\left(\phi(A)^{m-1} \phi(X)\right)+\operatorname{tr}\left(\phi(A)^{m-1} \phi(Y)\right) \\
& =(\phi(X) x, x)+(\phi(Y) x, x) .
\end{aligned}
$$

Since this is true for all unit vectors $x \in H$, it follows that $\phi(X+Y)=$ $\phi(X)+\phi(Y)$. Similarly, we can show that $\phi(\lambda X)=\lambda \phi(X)$ for all $\lambda \in \mathbb{F}$ and $X \in \mathbf{V}$.

It is well known that if $A \in M_{2}$ then $W(A)$ is an elliptical disk with the eigenvalues of $A$ as foci. Moreover, if $A \in B(H)$ is unitarily similar to $A_{1} \oplus A_{2}$ then $W(A)$ is the convex hull of $W\left(A_{1}\right) \cup W\left(A_{2}\right)$. In particular, if $A$ has rank one, then $A$ is unitarily similar to $C \oplus 0$, where $C$ has a matrix representation $\left(\begin{array}{ll}a & b \\ 0 & 0\end{array}\right)$; hence $W(A)=W(C)$ is an elliptical disk with 0 as a focus. These facts are used in the proof of the following lemma, which is an extension of a result in [5].

Lemma 2.2. Let $r$ and $s$ be two nonnegative integers with $r+s>0$. For any $B \in B(H), B$ has rank one if and only if for all $A \in B(H), W\left(A^{r} B A^{s}\right)$ is an elliptical disk with zero as one of the foci.

Proof. Let $B \in B(H)$. If $B$ is rank one, then so is $A^{r} B A^{s}$. Therefore $W\left(A^{r} B A^{s}\right)$ is an elliptical disk with 0 as a focus by the discussion before the lemma.

Conversely, suppose $B$ has rank at least 2. Then there exist $x, y \in H$ such that $\{B x, B y\}$ is an orthonormal set. Let $C=x(B x)^{*}-y(B y)^{*}$. Then $B C=B x(B x)^{*}-B y(B y)^{*}$ has numerical range $[-1,1]$. Suppose $r=0$. Since $C$ has rank two, it has an operator matrix of the form $C_{1} \oplus 0$, where $C_{1} \in M_{k}$ with $2 \leq k \leq 4$, with respect to an orthonormal basis of $H$. Let $D$ have operator matrix $\operatorname{diag}(1, \ldots, k) \oplus 0$ with respect to the same basis. Then $C+\nu D$ has operator matrix $\left(C_{1}+\nu D_{1}\right) \oplus 0$, where $D_{1}=\operatorname{diag}(1, \ldots, k)$. Except for finitely many $\nu \in \mathbb{R}, C_{1}+\nu D_{1}$ has distinct eigenvalues so that there is $A_{\nu}$ satisfying $A_{\nu}^{s}=C+\nu D$, and $W\left(B A_{\nu}^{s}\right)=W(B C+\nu B D)$. By [4, Problem 220], the mapping $\nu \mapsto \operatorname{Closure}(W(B C+\nu B D))$ is continuous. Since $W(B C)=[-1,1]$, there is a sufficiently small $\nu>0$ such that $W\left(B A_{\nu}^{s}\right)$ is not an elliptical disk with 0 as a focus. If $s=0$, we can fix an orthonormal basis of $H$, and apply the above argument to $B^{t}$ to show that there exists 
$A$ such that $W\left(A^{r} B\right)=W\left(B^{t}\left(A^{t}\right)^{r}\right)$ is not an elliptical disk with 0 as a focus.

Now, suppose that $r s>0$. Let $H_{0}$ be the subspace of $H$ spanned by $\{x, y, B x, B y\}$, which has dimension $p \in\{2,3,4\}$. Suppose $B_{0} \in M_{p}$ is the compression of $B$ on $H_{0}$. Then $B_{0}=P U$ for some positive semidefinite $P \in S_{p}$ with rank at least 2 , and a unitary matrix $U \in M_{p}$. Let $V \in M_{p}$ be a unitary matrix such that $V^{*} U V$ is in diagonal form. Then $V^{*} P V$ is positive semidefinite with rank at least 2 . Note that the $2 \times 2$ principal minors of $V^{*} P V$ are nonnegative, and their sum is the 2-elementary symmetric function of the eigenvalues of $V^{*} P V$, which is positive. So, at least one $2 \times 2$ principal minor of $V^{*} P V$ is nonzero. Since $V^{*} B_{0} V$ is the product of $V^{*} P V$ and the diagonal unitary matrix $V^{*} U V$, the $2 \times 2$ principal minors of $V^{*} B_{0} V$ are unit multiples of those of $V^{*} P V$. It follows that at least one $2 \times 2$ principal minor of $V^{*} B_{0} V$ is nonzero. Hence, there exists a two-dimensional subspace $H_{1}$ of $H_{0}$ such that the compression $B_{1}$ of $B$ on $H_{1}$ is invertible. Suppose $\{u, v\}$ is an orthonormal basis of $H_{1}$ such that $B_{1}=a u u^{*}+b u v^{*}+c v v^{*}$. Then $\operatorname{det}\left(B_{1}\right)=a c \neq 0$. Let $A=\alpha u u^{*}+\beta v v^{*}$ so that $\alpha^{r+s} a=1$ and $\beta^{r+s} c=-1$. Then $A^{r} B A^{s}=u u^{*}-v v^{*}+\alpha^{r} \beta^{s} b u v^{*}$ and $W\left(A^{r} B A^{s}\right)$ is an elliptical disk with foci $1,-1$.

Note that the analog of the above result for $\mathbf{V}=S(H)$ does not hold if $H$ has dimension at least 3. For example, if $A * B=A B A$ and $B=$ $u u^{*}+v v^{*}$ for some orthonormal set $\{u, v\}$ in $H$, then $W(A B A)$ is always a line segment with 0 as an end point. To prove our main theorems, we need a characterization of elements in $\mathcal{R}^{m}$ when $\mathbf{V}=S(H)$.

LEMMA 2.3. Let $r, s$ and $m$ be nonnegative integers such that $m-1=$ $r+s>0$. Suppose $X \in S(H)$ is such that $W\left(X^{m}\right)=[0,1]$. Then $X \in \mathcal{R}^{m}$ if and only if the following holds:

For any $Y \in S(H)$ satisfying $W\left(Y^{m}\right)=[0,1]=W\left(X^{r} Y X^{s}\right)$, we have

$$
\begin{aligned}
\{Z \in S(H): & \left.W\left(Z^{m}\right)=[0,1], Y^{r} Z Y^{s}=0_{H}\right\} \\
& \subseteq\left\{Z \in S(H): W\left(Z^{m}\right)=[0,1], X^{r} Z X^{s}=0_{H}\right\} .
\end{aligned}
$$

Proof. Since $W\left(X^{m}\right)=[0,1], X$ has an eigenvalue $\mu$ satisfying $\mu^{m}=1$ with a unit eigenvector $u$. Assume that $X \neq \mu u u^{*}$. Then $X=[\mu] \oplus X_{2}$ on $H=\operatorname{span}\{u\} \oplus\{u\}^{\perp}$, where $X_{2}$ is nonzero. Let $Y=[\mu] \oplus 0_{\{u\}^{\perp}}$. Then $W\left(Y^{m}\right)=[0,1]=W\left(X^{r} Y X^{s}\right)$. Note that the operator $Z=[0] \oplus I_{\{u\}^{\perp}}$ satisfies $W\left(Z^{m}\right)=[0,1]$ and $Y^{r} Z Y^{s}=0_{H}$ but $X^{r} Z X^{s}=[0] \oplus X_{2}^{m-1} \neq 0_{H}$.

Conversely, suppose $X=\mu u u^{*}$ on $H=\operatorname{span}\{u\} \oplus\{u\}^{\perp}$. For any $Y \in$ $S(H)$ satisfying $W\left(Y^{m}\right)=[0,1]=W\left(X^{r} Y X^{s}\right)$, we have $Y=[\mu] \oplus Y_{1}$ and 
$W\left(Y_{1}^{m}\right) \subseteq[0,1]$. Suppose

$$
Z=\left(\begin{array}{cc}
\alpha & z_{1}^{*} \\
z_{1} & Z_{2}
\end{array}\right)
$$

on $\operatorname{span}\{u\} \oplus\{u\}^{\perp}$ satisfying $W\left(Z^{m}\right)=[0,1]$ and $Y^{r} Z Y^{s}=0_{H}$. If $r s>0$ then $\alpha=0$; if $r s=0$ then $\alpha=0$ and $z_{1}=0$. In both cases, we see that $X^{r} Z X^{s}=0_{H}$.

\section{Proofs of the main theorems}

3.1. Proof of Theorem 1.2. We need the following lemma.

Lemma 3.1. Let $\mathbf{V}=M_{n}$ or $S_{n}$, and let $\phi: \mathbf{V} \rightarrow \mathbf{V}$ be a map satisfying (1.2). Then

$$
\phi\left(\mathcal{R}^{m}\right) \subseteq \mathcal{R}^{m}
$$

Proof. Each matrix $A \in \mathcal{R}^{m}$ can be written as $\mu U^{*} E_{11} U$ for some unitary matrix $U$ and $\mu \in \mathbb{F}$ with $\mu^{m}=1$. It suffices to prove that $\phi\left(E_{11}\right) \in \mathcal{R}^{m}$. For the other cases, we may replace the map $\phi$ by the map $A \mapsto \phi\left(\mu U^{*} A U\right)$.

We first consider the case when $\mathbf{V}=S_{n}$. For $i=1, \ldots, n$, let $F_{i}=\phi\left(E_{i i}\right)$. Since $E_{i i}^{r} E_{j j} E_{i i}^{s}=0_{n}$ for all $i \neq j$, we have

$$
W\left(F_{i}^{r} F_{j} F_{i}^{s}\right)=W\left(E_{i i}^{r} E_{j j} E_{i i}^{s}\right)=W\left(0_{n}\right)=\{0\} .
$$

It follows that $F_{i}^{r} F_{j} F_{i}^{s}=0_{n}$ for all $i \neq j$.

We claim that $F_{i} F_{j}=F_{j} F_{i}=0_{n}$ for all $i \neq j$. If the claim holds, then there are $\alpha_{1}, \ldots, \alpha_{n} \in \mathbb{R}$ and a unitary matrix $V$ such that $F_{i}=\alpha_{i} V^{*} E_{i i} V$. Furthermore, as $W\left(F_{i}^{m}\right)=W\left(E_{i i}^{m}\right)=[0,1], \alpha_{i}^{m}=1$. Therefore, $\phi\left(E_{11}\right)=$ $F_{1}=\alpha_{1} V^{*} E_{11} V \in \mathcal{R}^{m}$ and the result follows.

When $m$ is odd, as $W\left(\phi\left(I_{n}\right)^{m}\right)=W\left(I_{n}^{m}\right)=\{1\}$, we have $\phi\left(I_{n}\right)=I_{n}$. Then for any $i=1, \ldots, n$,

$$
W\left(F_{i}\right)=W\left(\phi\left(I_{n}\right)^{r} \phi\left(E_{i i}\right) \phi\left(I_{n}\right)^{s}\right)=W\left(I_{n}^{r} E_{i i} I_{n}^{s}\right)=W\left(E_{i i}\right)=[0,1] .
$$

Thus, $F_{i}$ is positive semidefinite. Now for any $i \neq j$, as $F_{i}^{r} F_{j} F_{i}^{s}=0_{n}$, we deduce that $F_{i} F_{j}=F_{j} F_{i}=0_{n}$.

When $m$ is even, since $W\left(\phi\left(I_{n}\right)^{m}\right)=\{1\}$, the eigenvalues of $\phi\left(I_{n}\right)$ can be either 1 or -1 only. Write $\phi\left(I_{n}\right)=V^{*}\left(I_{p} \oplus-I_{q}\right) V$ for some unitary matrix $V$ and nonnegative integers $p$ and $q$ such that $p+q=n$. Then for any $i=1, \ldots, n$,

$$
W\left(\phi\left(I_{n}\right)^{r} \phi\left(E_{i i}\right) \phi\left(I_{n}\right)^{s}\right)=W\left(I_{n}^{r} E_{i i} I_{n}^{s}\right)=W\left(E_{i i}\right)=[0,1] .
$$

Since one of $r$ and $s$ is odd while the other one must be even, either $\phi\left(I_{n}\right) F_{i}$ or $F_{i} \phi\left(I_{n}\right)$ is positive semidefinite. In both cases, we conclude that $F_{i}=V^{*}\left(P_{i} \oplus-Q_{i}\right) V$ for some positive semidefinite matrices $P_{i} \in H_{p}$ and $Q_{i} \in H_{q}$. Since $F_{i}^{r} F_{j} F_{i}^{s}=0_{n}$, we have $P_{i}^{r} P_{j} P_{i}^{s}=0_{p}$ and $Q_{i}^{r} Q_{j} Q_{i}^{s}=0_{q}$ for 
all $i \neq j$. Then we conclude that $P_{i} P_{j}=P_{j} P_{i}=0_{p}$ and $Q_{i} Q_{j}=Q_{j} Q_{i}=0_{q}$ and hence $F_{i} F_{j}=F_{j} F_{i}=0_{n}$.

So, our claim is proved and the lemma follows if $\mathbf{V}=S_{n}$.

Next, we turn to the case when $\mathbf{V}=M_{n}$. We divide the proof into a sequence of assertions. Then

Assertion 1. Let $D=\operatorname{diag}\left(0, e^{i \theta_{2}}, \ldots, e^{i \theta_{n}}\right)$ with $0<\theta_{2}<\cdots<\theta_{n}<\pi / m$.

$$
\phi(D)=V^{*}([0] \oplus T) V
$$

for some unitary matrix $V \in M_{n}$ and invertible upper triangular matrix $T \in M_{n-1}$.

Proof. Note that $D^{m}$ has $n$ distinct eigenvalues and $W\left(D^{m}\right)$ is a polygon with $n$ vertices including zero. Since $W\left(\phi(D)^{m}\right)=W\left(D^{m}\right)$, it follows that $\phi(D)^{m}$ has $n$ distinct eigenvalues, including one zero eigenvalue. Then so has $\phi(D)$. Therefore, we may write

$$
\phi(D)=V^{*}\left(\begin{array}{cc}
0 & x^{*} \\
0 & T
\end{array}\right) V
$$

for some $x \in \mathbb{C}^{n-1}$, a unitary matrix $V$ and an upper triangular matrix $T \in M_{n-1}$ such that all eigenvalues of $T$ are nonzero. Then $T$ is invertible. Since $W\left(\phi(D)^{m}\right)$ is a polygon with $n$ vertices, $\phi(D)^{m}$ is a normal matrix. Note that an upper triangular matrix is normal if and only if it is diagonal. Observe that

$$
\phi(D)^{m}=V^{*}\left(\begin{array}{cc}
0 & x^{*} T^{m-1} \\
0 & T^{m}
\end{array}\right) V .
$$

It follows that $x=0$ as $T$ is invertible, i.e., $\phi(D)=V^{*}([0] \oplus T) V$. The proof of the assertion is complete.

ASSERTION 2. The inclusion (3.1) holds if $r s=0$.

Proof. Suppose $r=0$. Then, as $E_{11} D^{s}=0_{n}$, where $D$ is the matrix defined in Assertion 1, $\phi\left(E_{11}\right) \phi(D)^{s}=0_{n}$. It follows that only the first column of $V^{*} \phi\left(E_{11}\right) V$ is nonzero, where $V$ is the unitary matrix defined in Assertion 1. Hence, $\phi\left(E_{11}\right)$ is a rank one matrix. Note that $W\left(\phi\left(E_{11}\right)^{m}\right)=$ $W\left(E_{11}^{m}\right)=[0,1]$, and since a rank one matrix $A \in M_{n}$ satisfies $W\left(A^{m}\right)=$ $[0,1]$ if and only if $A \in \mathcal{R}^{m}$, we conclude that $\phi\left(E_{11}\right) \in \mathcal{R}^{m}$. The proof for $s=0$ is similar. Thus, our assertion is true.

Assertion 3. Suppose $r s>0$. For any nonzero

$$
A=\left(\begin{array}{cc}
a & w^{*} \\
z & 0_{n-1}
\end{array}\right) \in M_{n},
$$


we have

$$
\phi\left(\left(\begin{array}{cc}
a & w^{*} \\
z & 0_{n-1}
\end{array}\right)\right)=V^{*}\left(\begin{array}{cc}
\alpha & x^{*} \\
y & 0_{n-1}
\end{array}\right) V
$$

for some $\alpha \in \mathbb{C}$ and $x, y \in \mathbb{C}^{n-1}$, where $V$ is the unitary matrix defined in Assertion 1. Furthermore, if $A^{m} \neq 0_{n}$ is Hermitian, then $x=\beta y$ for some nonzero $\beta \in \mathbb{C}$.

Proof. Let $D$ be the matrix defined in Assertion 1. Since $D^{r} A D^{s}=0_{n}$, it follows that $\phi(D)^{r} \phi(A) \phi(D)^{s}=0_{n}$. Thus

$$
\phi(A)=V^{*}\left(\begin{array}{cc}
\alpha & x^{*} \\
y & 0_{n-1}
\end{array}\right) V
$$

for some $\alpha \in \mathbb{C}$ and $x, y \in \mathbb{C}^{n-1}$, where $V$ is defined in Assertion 1. If $A^{m} \neq 0$ is Hermitian, $W\left(\phi(A)^{m}\right)=W\left(A^{m}\right) \subseteq \mathbb{R}$. Hence, $\phi(A)^{m}$ is Hermitian as well. Clearly, if $x$ or $y$ is the zero vector, say $x=0$, then $\alpha \neq 0$ as $A^{m} \neq 0_{n}$. Therefore, $y$ must also be zero. Thus the assertion holds.

Now we assume that both $x$ and $y$ are nonzero vectors. By induction, we have

$$
\phi(A)^{k}=V^{*}\left(\begin{array}{cc}
a_{k+1} & a_{k} x^{*} \\
a_{k} y & a_{k-1} y x^{*}
\end{array}\right) V \quad \text { for all } k=1,2, \ldots,
$$

where the sequence $\left\{a_{k}\right\}$ satisfies $a_{k+1}=\alpha a_{k}+x^{*} y a_{k-1}$ with $a_{0}=0, a_{1}=1$ and $a_{2}=\alpha$.

It is impossible to have both $a_{m}$ and $a_{m-1}$ equal to zero, since then $a_{m+1}=0$, and hence $\phi(A)^{m}=0_{n}$. Then $W\left(A^{m}\right)=W\left(\phi(A)^{m}\right)=\{0\}$, which contradicts our assumption that $A^{m} \neq 0_{n}$. Thus, $a_{m}$ or $a_{m-1}$ must be nonzero. In both cases, as $A^{m}$ is Hermitian, we must have $x=\beta y$ for some nonzero $\beta \in \mathbb{C}$. The proof of our assertion is complete.

Assertion 4. The inclusion (3.1) holds if $r s>0$.

Proof. For $i=1, \ldots, n$, let $H_{i}=\frac{1}{2}\left(E_{1 i}+E_{i 1}\right)$. Then $H_{i}^{m}$ is Hermitian and $H_{i}^{m} \neq 0_{n}$. By Assertion 3, we write

$$
\phi\left(H_{i}\right)=V^{*}\left(\begin{array}{cc}
\alpha_{i} & \beta_{i} z_{i}^{*} \\
z_{i} & 0_{n-1}
\end{array}\right) V
$$

for some $\alpha_{i}, \beta_{i} \in \mathbb{C}$ and $z_{i} \in \mathbb{C}^{n-1}$ with $\beta_{i} \neq 0$. Denote by $Z_{i}$ the $n \times 2$ matrix $\left(\begin{array}{ll}1 & 0 \\ 0 & z_{i}\end{array}\right)$ and by $K_{i}$ the $2 \times 2$ matrix $\left(\begin{array}{cc}\alpha_{i} & \beta_{i} \\ 1 & 0\end{array}\right)$. Then

$$
\phi\left(H_{i}\right)=V^{*}\left[\left(\begin{array}{cc}
1 & 0 \\
0 & z_{i}
\end{array}\right)\left(\begin{array}{cc}
\alpha_{i} & \beta_{i} \\
1 & 0
\end{array}\right)\left(\begin{array}{cc}
1 & 0 \\
0 & z_{i}^{*}
\end{array}\right)\right] V=V^{*} Z_{i} K_{i} Z_{i}^{*} V .
$$


Observe that for any distinct $i<j, H_{i}^{r} H_{j} H_{i}^{s}=0_{n}$. Setting $R_{i j}=Z_{i}^{*} Z_{j}$, we have

$$
\begin{aligned}
0_{n} & =\phi\left(H_{i}\right)^{r} \phi\left(H_{j}\right) \phi\left(H_{i}\right)^{s} \\
& =V^{*} Z_{i}\left(K_{i} R_{i i}\right)^{r-1} K_{i}\left[R_{i j} K_{j} R_{i j}^{*}\right] K_{i}\left(R_{i i} K_{i}\right)^{s-1} Z_{i}^{*} V .
\end{aligned}
$$

Next we claim that for any $1 \leq i<j \leq n$,

$$
z_{i}^{*} z_{j}=\alpha_{j}=0 \quad \text { and } \quad z_{j} \neq 0 \quad \text { whenever } z_{i} \neq 0 .
$$

To see this, suppose $z_{i} \neq 0$. Then the $n \times 2$ matrix $Z_{i}$ has rank 2 and hence the $2 \times 2$ matrix $Z_{i}^{*} Z_{i}$ is invertible. Also both $K_{i}$ and $K_{j}$ are invertible. Then (3.2) holds only when

$$
\left(\begin{array}{cc}
1 & 0 \\
0 & z_{i}^{*} z_{j}
\end{array}\right)\left(\begin{array}{cc}
\alpha_{j} & \beta_{j} \\
1 & 0
\end{array}\right)\left(\begin{array}{cc}
1 & 0 \\
0 & z_{j}^{*} z_{i}
\end{array}\right)=\left(Z_{i}^{*} Z_{j}\right) K_{j}\left(Z_{j}^{*} Z_{i}\right)=0_{2} .
$$

Thus, $\beta_{j} z_{j}^{*} z_{i}=z_{i}^{*} z_{j}=\alpha_{j}=0$. Finally, since $W\left(\phi\left(H_{j}\right)^{m}\right)=W\left(H_{j}^{m}\right) \neq\{0\}$, we have $z_{j} \neq 0$.

Now we must have $z_{1}=0$. Otherwise, $\alpha_{j}=z_{1}^{*} z_{j}=0$ and $z_{j} \neq 0$ for all $j=2, \ldots, n$. We can then further deduce that $z_{i}^{*} z_{j}=0$ for all $i \neq j$. Thus, we have $n$ nonzero orthogonal vectors $z_{1}, \ldots, z_{n}$ in $\mathbb{C}^{n-1}$, which is impossible. Therefore, $z_{1}=0$ and hence $\alpha_{1} \neq 0$. Finally, as $W\left(\phi\left(H_{1}\right)^{m}\right)=$ $W\left(H_{1}^{m}\right)=[0,1]$, we have $\alpha_{1}^{m}=1$. So $\phi\left(E_{11}\right)=\phi\left(H_{1}\right)=\alpha_{1} V^{*} E_{11} V \in \mathcal{R}^{m}$ and the result follows. The proof of our assertion is complete.

Combining the assertions, we get the result for $\mathbf{V}=M_{n}$.

Proof of Theorem 1.2. First, consider the sufficiency part. If (a) or (b) holds, then clearly $\phi$ satisfies (1.2). Suppose (c) holds. Then for any $A, B$ $\in S_{2}$, there is a unitary $V \in M_{2}$ such that $V^{*} A V=D$ is a real diagonal matrix, and $V^{*} B V=C$ is a real symmetric matrix. Thus,

$$
\begin{aligned}
\phi\left(A^{r} B A^{s}\right) & =W\left(D^{r} C D^{s}\right)=W\left(\overline{D^{r} C D^{s}}\right) \\
& =W\left(\left(D^{t}\right)^{r} C^{t}\left(D^{t}\right)^{s}\right)=W\left(\phi(A)^{r} \phi(B) \phi(A)^{s}\right) .
\end{aligned}
$$

Next we turn to the necessity. Suppose $\mathbf{V}=B(H)$ or $S(H)$. Assume that $\phi: \mathbf{V} \rightarrow \mathbf{V}$ satisfies (1.2), and that $\phi$ is surjective if $H$ is infinite-dimensional. We divide the proof into several steps.

STEP 1. We show that $\phi\left(\mathcal{R}^{m}\right)=\mathcal{R}^{m}$ and $\phi$ is linear. Suppose $H$ is finite-dimensional with no surjectivity assumption on $\phi$. By Lemma 3.1, $\phi\left(\mathcal{R}^{m}\right) \subseteq \mathcal{R}^{m}$. Suppose $H$ is infinite-dimensional. For $\mathbf{V}=S(H)$, we have $\phi\left(\mathcal{R}^{m}\right)=\mathcal{R}^{m}$ by Lemma 2.3 and the surjectivity of $\phi$. For $\mathbf{V}=B(H)$, by Lemma 2.2 and the surjectivity of $\phi$, we see that $\phi$ maps the set of rank one operators onto itself; since a rank one operator $A \in B(H)$ satisfies $W\left(A^{m}\right)=[0,1]$ if and only if $A \in \mathcal{R}^{m}$, we also have $\phi\left(\mathcal{R}^{m}\right)=\mathcal{R}^{m}$. 
Now, for any $A \in \mathcal{R}^{m}$ and $B \in \mathbf{V}$, both $A^{r} B A^{s}$ and $\phi(A)^{r} \phi(B) \phi(A)^{s}$ have rank at most one. As a result, $W\left(A^{r} B A^{s}\right)$ is an elliptical disk with foci $\operatorname{tr}\left(A^{r} B A^{s}\right)$ and 0 , and $W\left(\phi(A)^{r} \phi(B) \phi(A)^{s}\right)$ is an elliptical disk with foci $\operatorname{tr}\left(\phi(A)^{r} \phi(B) \phi(A)^{s}\right)$ and 0. Since $W\left(A^{r} B A^{s}\right)=W\left(\phi(A)^{r} \phi(B) \phi(A)^{s}\right)$, we conclude that

$$
\operatorname{tr}\left(A^{r+s} B\right)=\operatorname{tr}\left(A^{r} B A^{s}\right)=\operatorname{tr}\left(\phi(A)^{r} \phi(B) \phi(A)^{s}\right)=\operatorname{tr}\left(\phi(A)^{r+s} \phi(B)\right)
$$

for all $A \in \mathcal{R}^{m}$ and $B \in \mathbf{V}$. By Proposition 2.1, $\phi$ is linear. Moreover, if $H$ is finite-dimensional, $\phi$ is invertible. Indeed, $\phi^{-1}$ also satisfies (1.2), and hence (3.1) and (3.3). So, $\phi\left(\mathcal{R}^{m}\right)=\mathcal{R}^{m}$.

SteP 2. We show that $\phi\left(I_{H}\right)=\mu I_{H}$ with $\mu^{m}=1$. For any $x \in H$ with $(x, x)=1$, there are $y \in H$ and $\mu \in \mathbb{F}$ with $(y, y)=\mu^{m}=1$ such that $\phi\left(\mu y y^{*}\right)=x x^{*}$. Then by $(3.3)$,

$$
\begin{aligned}
\left(\phi\left(I_{H}\right) x, x\right) & =\operatorname{tr}\left(x x^{*} \phi\left(I_{H}\right)\right)=\operatorname{tr}\left(\left(x x^{*}\right)^{m-1} \phi\left(I_{H}\right)\right)=\operatorname{tr}\left(\phi\left(\mu y y^{*}\right)^{m-1} \phi\left(I_{H}\right)\right) \\
& =\operatorname{tr}\left(\left(\mu y y^{*}\right)^{m-1} I_{H}\right)=\mu^{m-1}(y, y)=\mu^{-1} .
\end{aligned}
$$

It follows that $W\left(\phi\left(I_{H}\right)\right) \subseteq\left\{\mu^{-1}: \mu^{m}=1\right\}=\left\{\mu: \mu^{m}=1\right\}$. By the convexity of numerical range, $W\left(\phi\left(I_{H}\right)\right)$ is a singleton set. Thus, $\phi\left(I_{H}\right)=$ $\mu I_{H}$ for some $\mu^{m}=1$.

STEP 3. We show that $\phi$ has the asserted form. Using the result in Step 2, and replacing $\phi$ by the map $A \mapsto \mu^{-1} \phi(A)$, we have $\phi\left(I_{H}\right)=I_{H}$. Furthermore,

$W(\phi(A))=W\left(\phi\left(I_{H}\right)^{r} \phi(A) \phi\left(I_{H}\right)^{s}\right)=W\left(I_{H}^{r} A I_{H}^{s}\right)=W(A) \quad$ for all $A \in \mathbf{V}$.

Since $\phi$ is linear, by the results in $[7,8]$ the map $\phi$ has the form

$$
A \mapsto U^{*} A U \quad \text { or } \quad A \mapsto U^{*} A^{t} U
$$

for some unitary operator $W \in B(H)$.

STEP 4. It remains to show that $r=s$ when $\mathbf{V} \neq S_{2}$ and $\phi$ has the form $A \mapsto U^{*} A^{t} U$. For any $A, B \in \mathbf{V}$,

$$
\begin{aligned}
W\left(A^{s} B A^{r}\right) & =W\left(\left(A^{t}\right)^{r} B^{t}\left(A^{t}\right)^{s}\right)=W\left(U^{*}\left(A^{t}\right)^{r} B^{t}\left(A^{t}\right)^{s} U\right) \\
& =W\left(\phi(A)^{r} \phi(B) \phi(A)^{s}\right)=W\left(A^{r} B A^{s}\right) .
\end{aligned}
$$

For $\mathbf{V}=B(H)$, let $\{u, v\}$ be an orthonormal set in $H, A=u u^{*}+u v^{*}+v v^{*}$ and $B=v v^{*}$. Then

$$
W\left(s u v^{*}+v v^{*}\right)=W\left(A^{s} B A^{r}\right)=W\left(A^{r} B A^{s}\right)=W\left(r u v^{*}+v v^{*}\right) .
$$

Thus, $r=s$ and the result follows.

Now consider $\mathbf{V}=S(H)$, where $H$ has dimension at least 3. Suppose $r \neq s$. Without loss of generality, we assume that $r>s$. Let $A, B \in S(H)$ be such that

$$
A^{r-s}=D \oplus 0 \quad \text { and } \quad A^{s} B A^{s}=E \oplus 0,
$$


where

$$
D=\operatorname{diag}(3,2,1) \quad \text { and } \quad E=\left(\begin{array}{ccc}
1 & 1 & i \\
1 & 0 & 1 \\
-i & 1 & 0
\end{array}\right)
$$

with respect to a suitable orthonormal basis. Then

$$
\begin{aligned}
W(D E \oplus 0) & =W\left(A^{r} B A^{s}\right)=W\left(A^{s} B A^{r}\right)=W(E D \oplus 0) \\
& =W(\overline{D E \oplus 0})=\overline{W(D E \oplus 0)} .
\end{aligned}
$$

Therefore, $W(D E \oplus 0)$ is symmetric about the real axis. But this is impossible as the eigenvalues of $D E-E D$ are $2 i,(\sqrt{3}-1) i / 2$ and $(-\sqrt{3}-1) i / 2$. Hence $\left\{\operatorname{Im} z: z \in W\left(D E \oplus 0_{n-3}\right)\right\}=[(-\sqrt{3}-1) / 2,2]$ so that the two horizontal support lines of $W(D E \oplus 0)$ are $\{z: \operatorname{Im} z=2\}$ and $\{z: \operatorname{Im} z=$ $(-\sqrt{3}-1) / 2\}$, which is a contradiction. Therefore, we must have $r=s$.

The proof of our theorem is complete.

3.2. Proof of Theorem 1.1. If (a) holds then $\phi$ clearly satisfies (1.1). Suppose (b) holds. Then for any $A_{1}, \ldots, A_{k} \in \mathbf{V}$, we have

$$
\begin{aligned}
& W\left(\phi\left(A_{1}\right) * \cdots * \phi\left(A_{k}\right)\right) \\
& =W\left(\phi\left(A_{i_{1}}\right) \cdots \phi\left(A_{i_{m}}\right)\right)=W\left(U^{*} A_{i_{1}}^{t} \cdots A_{i_{m}}^{t} U\right)=W\left(\left(A_{i_{m}} \cdots A_{i_{1}}\right)^{t}\right) \\
& =W\left(A_{i_{m}} \cdots A_{i_{1}}\right)=W\left(A_{i_{1}} \cdots A_{i_{m}}\right)=W\left(A_{1} * \cdots * A_{k}\right) .
\end{aligned}
$$

Suppose (c) holds. Note that $X, Y \in M_{2}$ have the same numerical range if and only if the two matrices have the same eigenvalues and the same Frobenius norm, equivalently, $\operatorname{tr}(X)=\operatorname{tr}(Y)$, $\operatorname{det}(X)=\operatorname{det}(Y)$ and $\operatorname{tr}\left(X X^{*}\right)=$ $\operatorname{tr}\left(Y Y^{*}\right)$. One readily checks that these conditions are satisfied for $X=$ $A_{1} * \cdots * A_{k}$ and $Y=\phi\left(A_{1}\right) * \cdots * \phi\left(A_{k}\right)$ for any $A_{1}, \ldots, A_{k} \in S_{2}$ if (c) holds. So, condition (1.1) follows.

Next, we turn to the necessity. Applying Theorem 1.2 with $A_{i_{r}}=B$ and $A_{i_{s}}=A$ for all other $s \neq r$, we conclude that there exist a unitary operator $U \in B(H)$ and a scalar $\mu \in \mathbb{F}$ with $\mu^{m}=1$ such that one of the following holds:

(a) $A \mapsto \mu U^{*} A U$ for all $A \in \mathbf{V}$.

(b) $r=(m+1) / 2$ and $\phi$ has the form $A \mapsto \mu U^{*} A^{t} U$.

(c) $\mathbf{V}=S_{2}$ and $\phi$ has the form $A \mapsto \mu U^{*} A^{t} U$.

It remains to prove $\left(i_{r+1}, \ldots, i_{m}, i_{1}, \ldots, i_{r-1}\right)=\left(i_{r-1}, \ldots, i_{1}, i_{m}, \ldots, i_{r+1}\right)$ if (b) or (c) holds.

Evidently, the result holds for $k=2$ as we must have $i_{1}=\cdots=i_{r-1}=$ $i_{r+1}=\cdots=i_{m}$ in this case. Now we assume that $k \geq 3$. Then we have

$$
\begin{aligned}
W\left(A_{i_{1}} \cdots A_{i_{m}}\right) & =W\left(\phi\left(A_{i_{1}}\right) \cdots \phi\left(A_{i_{m}}\right)\right)=W\left(U^{*} A_{i_{1}}^{t} \cdots A_{i_{m}}^{t} U\right) \\
& =W\left(A_{i_{1}}^{t} \cdots A_{i_{m}}^{t}\right)=W\left(A_{i_{m}} \cdots A_{i_{1}}\right) .
\end{aligned}
$$


By taking $A_{i_{r}}=R$, where $R$ is a Hermitian rank one idempotent, and considering the foci of the elliptical disks for the above numerical ranges, we conclude that

$$
\begin{aligned}
\operatorname{tr}\left(A_{i_{r+1}} \cdots A_{i_{m}} A_{i_{1}} \cdots A_{i_{r-1}} R\right) & =\operatorname{tr}\left(A_{i_{1}} \cdots A_{i_{r-1}} R A_{i_{r+1}} \cdots A_{i_{m}}\right) \\
& =\operatorname{tr}\left(A_{i_{m}} \cdots A_{i_{r+1}} R A_{i_{r-1}} \cdots A_{i_{1}}\right) \\
& =\operatorname{tr}\left(A_{i_{r-1}} \cdots A_{i_{1}} A_{i_{m}} \cdots A_{i_{r+1}} R\right)
\end{aligned}
$$

Since $R$ can be an arbitrary Hermitian rank one idempotent, by the fact that $X$ and $Y$ are equal if $\operatorname{tr}(X R)=\operatorname{tr}(Y R)$ for all Hermitian rank one idempotent $R$, we deduce that

$$
A_{i_{r+1}} \cdots A_{i_{m}} A_{i_{1}} \cdots A_{i_{r-1}}=A_{i_{r-1}} \cdots A_{i_{1}} A_{i_{m}} \cdots A_{i_{r+1}}
$$

for all choices of $A_{1}, \ldots, A_{k}$.

We now use a similar argument to the one in the proof of $[1$, Theorem 2.1]. We give the details for the sake of completeness. For simplicity, we rename $\left(i_{r+1}, \ldots, i_{m}, i_{1}, \ldots, i_{r-1}\right)$ as $\left(j_{1}, \ldots, j_{m-1}\right)$ and we have to show that $\left(j_{1}, \ldots, j_{m-1}\right)=\left(j_{m-1}, \ldots, j_{1}\right)$. Suppose $\left(j_{1}, \ldots, j_{m-1}\right) \neq\left(j_{m-1}, \ldots, j_{1}\right)$. Let $1 \leq p \leq m / 2$ be the smallest integer such that $j_{p} \neq j_{m-p}$. For any $\lambda>0$, let $D=\operatorname{diag}(\lambda, 1)$ and $S$ be some $2 \times 2$ symmetric matrix with positive entries. Fix a two-dimensional subspace $H_{1}$ in $H$ and take $A_{j_{p}}=D \oplus I_{H_{1}^{\perp}}$ and $A_{j_{t}}=S \oplus I_{H_{1}^{\perp}}$ for all other $j_{t} \neq j_{p}$ on $H=H_{1} \oplus H_{1}^{\perp}$. Then

$$
A_{j_{p}} \cdots A_{j_{m-p}}=\left(D^{d_{1}} S^{s_{1}} D^{d_{2}} S^{s_{2}} \cdots D^{d_{q}} S^{s_{q}}\right) \oplus I_{H_{1}^{\perp}}
$$

for positive integers $d_{i}, s_{i}$. Note that

$$
D^{d_{i}} S^{s_{i}}=\left(\begin{array}{cc}
\lambda^{d_{i}} e_{i} & \lambda^{d_{i}} f_{i} \\
g_{i} & h_{i}
\end{array}\right) \text { and } S^{s_{i}} D^{d_{i}}=\left(\begin{array}{ccc}
\lambda^{d_{i}} e_{i} & f_{i} \\
\lambda^{d_{i}} g_{i} & h_{i}
\end{array}\right)
$$

for some positive numbers $e_{i}, f_{i}, g_{i}, h_{i}$. We check that the $(1,2)$ entry of $D^{d_{1}} S^{s_{1}} \cdots D^{d_{q}} S^{s_{q}}$ is a polynomial of degree $d_{1}+\cdots+d_{q}$ in $\lambda$, while the $(1,2)$ entry of $S^{s_{q}} D^{d_{q}} \cdots S^{s_{1}} D^{d_{1}}$ is a polynomial of degree $d_{2}+\cdots+d_{q}$. So, there is $\lambda>0$ such that

$$
\begin{aligned}
A_{j_{p}} \cdots A_{j_{m-p}} & =\left(D^{d_{1}} S^{s_{1}} \cdots D^{d_{q}} S^{s_{q}}\right) \oplus I_{H_{1}^{\perp}} \\
& \neq\left(S^{s_{q}} D^{d_{q}} \cdots S^{s_{1}} D^{d_{1}}\right) \oplus I_{H_{1}^{\perp}}=A_{j_{m-p}} \cdots A_{j_{p}} .
\end{aligned}
$$

It follows that $A_{j_{1}} \cdots A_{j_{m-1}} \neq A_{j_{m-1}} \cdots A_{j_{1}}$, which is a contradiction. Hence, $\left(j_{1}, \ldots, j_{m-1}\right)=\left(j_{m-1}, \ldots, j_{1}\right)$ as asserted.

Acknowledgements. We thank Professor Jinchuan Hou for sending us the preprint [5], and his inspiring presentation of the paper at the workshop on preserver problems at the University of Hong Kong in 2004. Thanks are also due to Dr. Jor-Ting Chan for his effort in organizing the two workshops on preserver problems at the University of Hong Kong in 2004 and 2005 that facilitated this research. 


\section{References}

[1] J. T. Chan, C. K. Li and N. S. Sze, Mappings preserving spectra of products of matrices, to appear in Proc. Amer. Math. Soc. preprint available at http://www.resnet.wm. edu/ cklixx/spectrum.pdf.

[2] W. S. Cheung, S. Fallat and C. K. Li, Multiplicative preservers on semigroups of matrices, Linear Algebra Appl. 355 (2002), 173-186.

[3] H. L. Gau and C. K. Li, $C^{*}$-isomorphisms, Jordan isomorphisms, and numerical range preserving maps, submitted.

[4] P. Halmos, A Hilbert Space Problem Book, 2nd ed., Springer, New York, 1982.

[5] J. Hou and Q. Di, Maps preserving numerical ranges of operator products, Proc. Amer. Math. Soc. 134 (2006), 1435-1446.

[6] G. Lešnjak, Additive preservers of numerical range, Linear Algebra Appl. 345 (2002), 235-253.

[7] C. K. Li, L. Rodman and P. Šemrl, Linear maps on selfadjoint operators preserving invertibility, positive definiteness, numerical range, Canad. Math. Bull. 46 (2003), 216-228.

[8] V. Pellegrini, Numerical range preserving operators on a Banach algebra, Studia Math. 54 (1975), 143-147.

Department of Mathematics

College of William and Mary

Williamsburg, VA 23185, U.S.A.

E-mail: ckli@math.wm.edu
Department of Mathematics University of Hong Kong

Hong Kong

E-mail: NungSingSze@graduate.hku.hk

Received July 18, 2005

Revised version December 2, 2005 